\title{
Acute Painful Ptosis Secondary to IgG4 Dacryoadenitis
}

\author{
Rumana Hussain Abdul El-Khyat Antonella Berry-Brincat \\ Department of Ophthalmology, Leicester Royal Infirmary, Leicester, UK
}

\section{Key Words}

IgG4 - Dacryoadenitis · Third nerve palsy

\begin{abstract}
A 48-year-old lorry driver presented with 3 weeks of blurred vision, pain and diplopia. There was a right upper lid ptosis with some restriction of eye movements. A CT revealed an enlarged lacrimal gland and lacrimal gland biopsy showed IgG4-positive plasma cells. The patient responded to oral prednisolone and fully recovered. As a condition which mimics a number of diseases, an IgG4-related disease presents a diagnostic challenge and ought to be considered in both acute and chronic presentations.

(c) 2016 The Author(s)

Published by S. Karger AG, Basel
\end{abstract}

\section{Introduction}

IgG4 dacryoadenitis forms a subcategory of conditions previously diagnosed as idiopathic orbital inflammation. The course of the disease is often insidious and a histological diagnosis is therefore often delayed. We report a case of IgG4 dacryoadenitis presenting atypically in the acute setting, where prompt diagnosis and treatment had a beneficial outcome.

\section{Case Presentation}

A 48-year-old lorry driver presented with a 3-week history of blurred vision, eye pain and binocular vertical diplopia associated with mild headache but was otherwise well. His medical history includes controlled hypertension.

On examination, visual acuity was logMAR 0.0 in both eyes, ocular examination was normal with no evidence of redness or inflammation. There was a right 2-mm upper lid pto-

\section{KARGER}

Rumana Hussain

Department of Ophthalmology, Leicester Royal Infirmary

Infirmary Square

Leicester LE1 5WW (UK)

E-Mail rumanahussain@hotmail.com 
Hussain et al.: Acute Painful Ptosis Secondary to IgG4 Dacryoadenitis

sis with suspected mild anisocoria (fig. 1a), with a slightly larger right pupil and restriction of the right eye in upgaze. There was no evidence of lymphadenopathy. A CT angiogram of the head and neck did not reveal any intracranial abnormality nor any signs of lymphadenopathy or salivary gland involvement; however, it demonstrated right lacrimal gland enlargement (fig. 1b). There was also evidence of nasal sinus congestion.

Blood tests for inflammatory, infectious and autoimmune markers as well as serum IgG4 did not reveal any abnormality. A lacrimal gland biopsy was performed the following week; the gland was noted to be firm and sclerosed. Immunohistochemistry showed IgG4-positive plasma cells and marked collagen sclerosis (fig. 1c-e). The patient was initiated on a tapering dose of $80 \mathrm{mg}$ oral prednisolone over 4 months with rapid resolution of symptoms and signs. At the 24-month follow-up, there were no signs of relapse or evidence of systemic organ involvement.

\section{Discussion}

IgG4 dacryoadenitis comprises a subgroup of patients previously diagnosed with idiopathic orbital inflammation. The disease is often chronic and indolent in nature with a history of eyelid swelling or proptosis, the majority occurring in male patients aged 21-79 years [1].

Although first described affecting the pancreas, the list of organs involved has rapidly expanded [2], predominantly causing pancreatitis, sclerosing cholangitis, retroperitoneal fibrosis and chronic sclerosing sialadenitis $[3,4]$. Other head and neck structures reported by IgG4 to be affected are thyroid gland, nasal sinuses, lymph nodes and larynx with similar infiltrative processes and the formation of mass-like lesions [5]. The organs affected by IgG4 are often observed sequentially, sometimes over several years, making full recognition of this disorder challenging. However, the disease may remain localized to one site [2], as in our patient with sole involvement of the lacrimal gland.

Although serum IgG4 levels may be utilised in the initial assessment of these cases, the results are variable; lower serum IgG4 levels have been suggested to be associated with reduced risk of bilateral or systemic disease [1], and lower rates of relapse following treatment [6].

The diagnosis is therefore based on histological analysis; however, biopsy often occurs late in typical patients with indolent symptoms. Histology characteristically comprises dense lymphoplasmic infiltration and storiform fibrosis and marked infiltration with IgG4-positive plasma cells ( $>10 /$ high power field and/or a ratio of IgG4-/IgG-positive cells $>40 \%$ ); obliterative phlebitis is infrequent in lacrimal gland specimens differentiating it from extranodal lymphoma.

The mainstay of IgG4-related disease treatment remains tapering doses of glucocorticoids; orbit-involving disease has been treated with corticosteroids; however, a metaanalysis suggests an unsustained response with relapse after treatment cessation [7]. There is no consensus as to whether pulsed steroids result in better outcomes than oral glucocorticoids alone $[2,8]$.

Some patients seem to remain stable without treatment [9], which may be related to normal baseline serum IgG4 levels [10]. Immunosuppressive agents such as azathioprine and mycophenolate mofetil [11], as well as biologic agents such as rituximab [12], and radiotherapy have been utilised in systemic disease as well as orbit-isolated disease unresponsive to initial therapy $[9,13]$. It has been postulated that sustained IgG4-related inflammation may evolve from an autoimmune to a chronic process resulting in expansive fibrosis and 
Hussain et al.: Acute Painful Ptosis Secondary to IgG4 Dacryoadenitis

cumulative organ damage; early treatment has therefore been proposed to prevent relapse following cessation of therapy [14]. Long-term observation is also warranted as there is emerging evidence of increased risk of lymphoma development $[2,15]$.

The current case presented atypically in the acute setting with features initially mimicking a neurological defect; prompt imaging, biopsy and treatment enabled a rapid-sustained and complete recovery. The double vision resolved as the inflammatory process settled, suggesting that the defects in extraocular movements were due to mechanical restriction, although concurrent myositis may be responsible (not evident on CT). The normal serum IgG4 level suggests that this is unreliable for diagnostic exclusion and therefore a histological sample is required. However, the continued absence of systemic disease on follow-up suggests that normal serum IgG4 may be a good prognostic sign.

\section{Statement of Ethics}

The authors have no ethical conflicts to disclose.

\section{Disclosure Statement}

The authors have no conflicts of interest to disclose.

\section{References}

1 Wallace ZS, Deshpande V, Stone JH: Ophthalmic manifestations of IgG4-related disease: single-center experience and literature review. Sem Arthritis Rheum 2014;43:806-817.

-2 Berry-Brincat A, Rose G: Idiopathic orbital inflammation: a new dimension with the discovery of immunoglobulin G4-related disease. Curr Opin Ophthalmol 2012;23:415-419.

-3 Hamano H, Kawa S, Ochi Y, Unno H, Shiba N, Wajiki M, et al: Hydronephrosis associated with retroperitoneal fibrosis and sclerosing pancreatitis. Lancet 2002;359:1403-1404.

4 Kitagawa S, Zen Y, Harada K, Sasaki M, Sato Y, Minato H, et al: Abundant IgG4-positive plasma cell infiltration characterizes chronic sclerosing sialadenitis (Kuttner's tumor). Am J Surg Pathol 2005;29:783791.

5 Fujita A, Sakai O, Chapman M, Sugimoto H: IgG4-related disease of the head and neck: CT and MR imaging manifestations. Radiographics 2012;32:1945-1958.

6 Kamisawa T, Chung JB, Irie H, Nishino T, Ueki T, Takase M, et al: Japan-Korea symposium on autoimmune pancreatitis (KOKURA 2007). Pancreas 2007;35:281-284.

7 Andrew N, Kearney D, Selva D: IgG4-related orbital disease: a meta-analysis and review. Acta Ophthalmol 2013;91:694-700.

8 Ghazale A, Chari ST: Optimising corticosteroid treatment for autoimmune pancreatitis. Gut 2007;56:16501652.

9 Sato Y, Ohshima K, Ichimura K, et al: Ocular adenexal IgG4 related disease has uniform clinicopathology. Pathol Int 2008;58:465-470.

$\checkmark 10$ Kubota K, Fujisawa T, et al: Clinical factors predictive of spontaneous remission in cases of autoimmune pancreatitis. Gastrointest Endosc 2007;66:1142-1151.

11 Kamisawa T, Shimosegawa T, Okazaki K, Nishino T, Watanabe H, Kanno A, et al: Standard steroid treatment for autoimmune pancreatitis. Gut 2009;58:1504-1507.

12 Ghazale A, Chari ST, Zhang L, Smyrk TC, Takahashi N, Levy MJ, et al: Immunoglobulin G4-associated cholangitis: clinical profile and response to therapy. Gastroenterology 2008;134:706-715.

13 Khosroshahi A, Carruthers MN, Deshpande V, et al: Rituximab for the treatment of IgG4 related disease: lessons learned form 10 consecutive patients. Medicine 2012;91:57-66.

14 Takahashi H, Yamamoto M, Shinomura Y, Imai K: Long-term outcomes and necessity of early intervention for IgG4-related disease. Nihon Rinsho Meneki Gakkai Kaishi 2013;36:442-451.

15 Mulay K, Aggarwal E: IgG4-related dacryoadenitis evolving into an extra-nodal, marginal zone B-cell lymphoma (EMZL): a tale of two lacrimal glands. Pathology 2014;46:464-466. 
Hussain et al.: Acute Painful Ptosis Secondary to IgG4 Dacryoadenitis
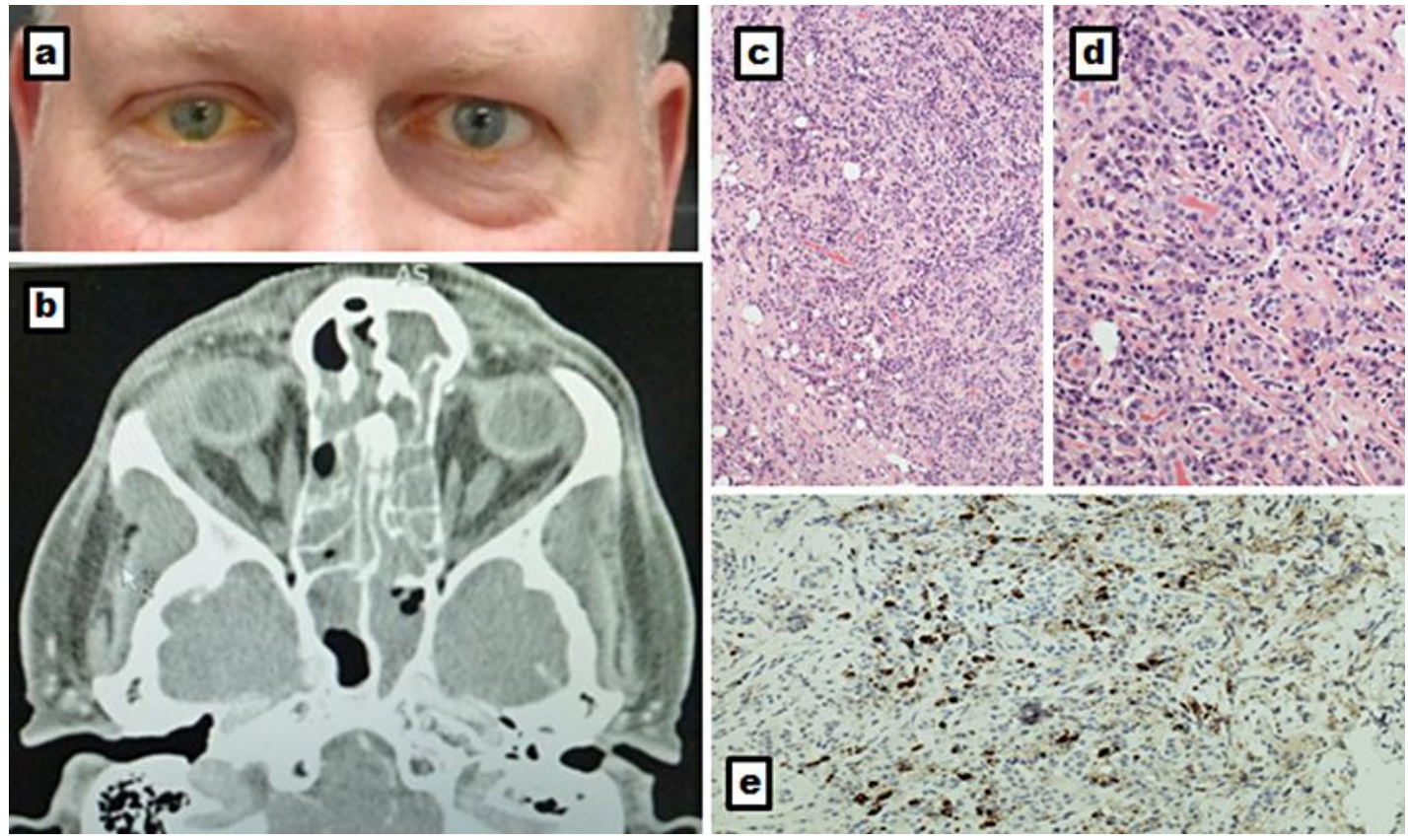

Fig. 1. a Acute presentation with a right partial ptosis. b Axial CT scan image showing an enlarged right lacrimal gland with no bony changes. c, $\mathbf{d}$ Haematoxylin and eosin staining of right lacrimal gland biopsy ( $\times 20$ and $\times 40$ magnification, respectively) showing destruction of lacrimal gland tissue with some duct preservation, extensive fibrosis and lymphoplasmocytic infiltration. e Immunoperoxidase staining $(\times 40$ magnification) showing IgG4-positive plasma cells. 